\title{
EXPERIMENTAL STUDIES OF A CERN-CLIC 32.98 GHZ HIGH GRADIENT ACCELERATING STRUCTURE DRIVEN BY THE MIT FREE ELECTRON LASER AMPLIFIER*
}

\author{
P. Volfbeyn, I. Mastovsky, G. Bekefi, MIT \\ I. Wilson, W. Wuensch, CERN-CLIC
}

Experimental studies on a CERN-CLIC $32.98 \mathrm{GHz} 26$-cell High Gradient Accelerating (HGA) section were carried out with input powers from $0.1 \mathrm{MW}$ to $50 \mathrm{MW}$. MIT's Free Electron Laser (FEL) amplifier driven by a mildly relativistic electron beam $(750 \mathrm{kV}, 300 \mathrm{~A}, 30 \mathrm{~ns})$ served as the RF power source for the structure. The maximum power in the transmitted pulse was measured to be $15 \mathrm{MW}$ for an input pulse of $\sim 35 \mathrm{MW}$. The theoretically calculated shunt impedance of $116 \mathrm{M} \Omega / \mathrm{m}$ predicts a field gradient of 65 $\mathrm{MeV} / \mathrm{m}$ inside the HGA. For power levels $>3 \mathrm{MW}$ the pulse transmitted through the HGA was observed to be shorter than the input pulse and pulse shortening became more serious with increasing power input. At the highest power levels the output pulse length (about $5 \mathrm{nsec}$ ) was about one quarter of the input pulse length. Various tests suggest that these undesirable effects occur in the input coupler to the HGA. Light and X-ray production inside the HGA have been observed. A feasibility study suggests that conditioning is a likely candidate for improving the performance of the HGA.

\section{INTRODUCTION}

The two-beam accelerator [1] is a promising candidate for achieving the ultra-high electron energies (of the order of or higher than $1 \mathrm{TeV}$ ) required in the next generation linear colliders. The HGA is a prototype of a section of the accelerating line of a two-beam accelerator.

For driving the HGA we used the MIT FEL [2,3] It produces $60 \mathrm{MW}$ r.f. power, $18 \mathrm{~ns}$ long pulses. A high power magnetron is the input power source for the FEL amplifier. It essentially determines the tunability of the FEL. The operating frequency range of the magnetron is from 30 to $35 \mathrm{GHz}$. The frequency of the magnetron pulse is stable to within at least $5 \mathrm{MHz}$. However the frequency shift induced by the FEL interaction could be of some concern. In the FEL used in the tests the frequency shift is measured to be $16 \mathrm{MHz}$ with $5 \mathrm{MHz}$ scatter in the Reversed Field regime [3]. The bandwidth of the HGA is greater than $500 \mathrm{MHz}$. Thus the MIT's FEL is acceptable for the purposes of the experiments described in this paper. For actual acceleration however, where the phase stability is of much greater importance, a phase stability better by one order of magnitude (less than $1 \mathrm{MHz}$ ) would be needed.

The $32.98 \mathrm{GHz}$ disc-loaded constant gradient traveling-wave accelerating structure was built as prototype of an element in the accelerating line of the two-beam
CERN-CLIC accelerator $[4,5]$. It is a $2 \pi / 3$ mode, $\mathrm{v}_{\mathrm{p}}=\mathrm{c}$, constant gradient iris disc-loaded waveguide structure. It consists of 26 cells, each of $3.029 \mathrm{~mm}$ in length and 2 coupler cells $3.56 \mathrm{~mm}$ long. The total length of the structure is $0.08587 \mathrm{~m}$. The fill time of the structure is $3.42 \mathrm{~ns}$, with a group velocity of $0.083 \mathrm{c}$. The two side-couplers are at right angles to the axis of the structure. They couple the power from a standard WR-28 rectangular waveguide into the desired mode of the structure. The high vacuum of around $10^{-7}$ Torr is maintained by two 30 liters/sec ion-pumps and two smaller auxilliary ion pumps with pumping speed of 2 liters/sec. The structure was designed to have shunt impedance of $116 \mathrm{M} \Omega / \mathrm{m}$. This means that for input power level of $60 \mathrm{MW}$ the maximum accelerating gradients approximately $110 \mathrm{MV} / \mathrm{m}$. The CERN-CLIC structure was designed for accelerating gradients of approximately 80 $\mathrm{MV} / \mathrm{m}$.

\section{EXPERIMENTAL RESULTS}

\section{A. High Power Testing}

With use of crystal detectors and variable attenuators attached to the ports of the high vacuum directional couplers it was possible to sample the time profile of a radiation pulse input into the HGA, transmitted through the HGA, and reflected back by the HGA. A scan in the input power level ranging from $0.1 \mathrm{MW}$ to about 35 MW was taken.

At low powers the transmitted pulse resembled the input pulse in shape and the percentage difference in power magnitudes was equal to the 'cold' attenuation as measured with a network analyzer (with very small $\sim 1 \mathrm{~mW}$ power levels). As the power levels increased $(\sim 3 \mathrm{MW})$ the transmitted power pulse became shorter than the input power pulse in time duration. The early part of the transmitted power pulse still resembled the input pulse, while the transmitted power was attenuated stronger and stronger the closer it approached the end of the pulse. The transmitted power 'rolled off' at the end of the pulse. At power levels from $20 \mathrm{MW}$ and higher the transmitted power pulse shape became effectively independent of the input pulse shape. The transmitted pulse peak power value was no longer proportional to that of the input pulse. With the increase of the input power, the transmitted power pulse saturated at a level of $\sim 14 \mathrm{MW}$. The reflected power rose to a maximum of about $6 \mathrm{MW}$. The plot of the FWHM of the transmitted pulse vs. the average power in the input pulse is

* Work supported by the U.S. Department of Energy,

Division of Nuclear and High Energy Physics 
shown in Figure 1. The fraction of the power in the reflected pulse would be expected to grow as the input power level increased, because the amount of the input power transmitted through the HGA was seen to decrease with increasing input power levels. It turned out to be only partly true. Power levels of the reflected pulse agreed with the cold test results when the input power level was small. The reflected power did grow to become comparable to the input power at high input power levels. But this growth did not fully account for the difference in energy between the input and transmitted pulses. A representation of our data is in terms of the energy in the pulse obtained by integrating the measured power over the respective pulse widths. In Figure 1 we present the dependence of the transmitted and the reflected energies on the average power in the input pulse. Note that at high input energies (corresponding to power levels of $20 \mathrm{MW}$ ) the transmitted and reflected energies together account for only a half of the input energy. This leads to the conclusion that a substantial fraction of the radiation energy is dissipated inside the HGA.
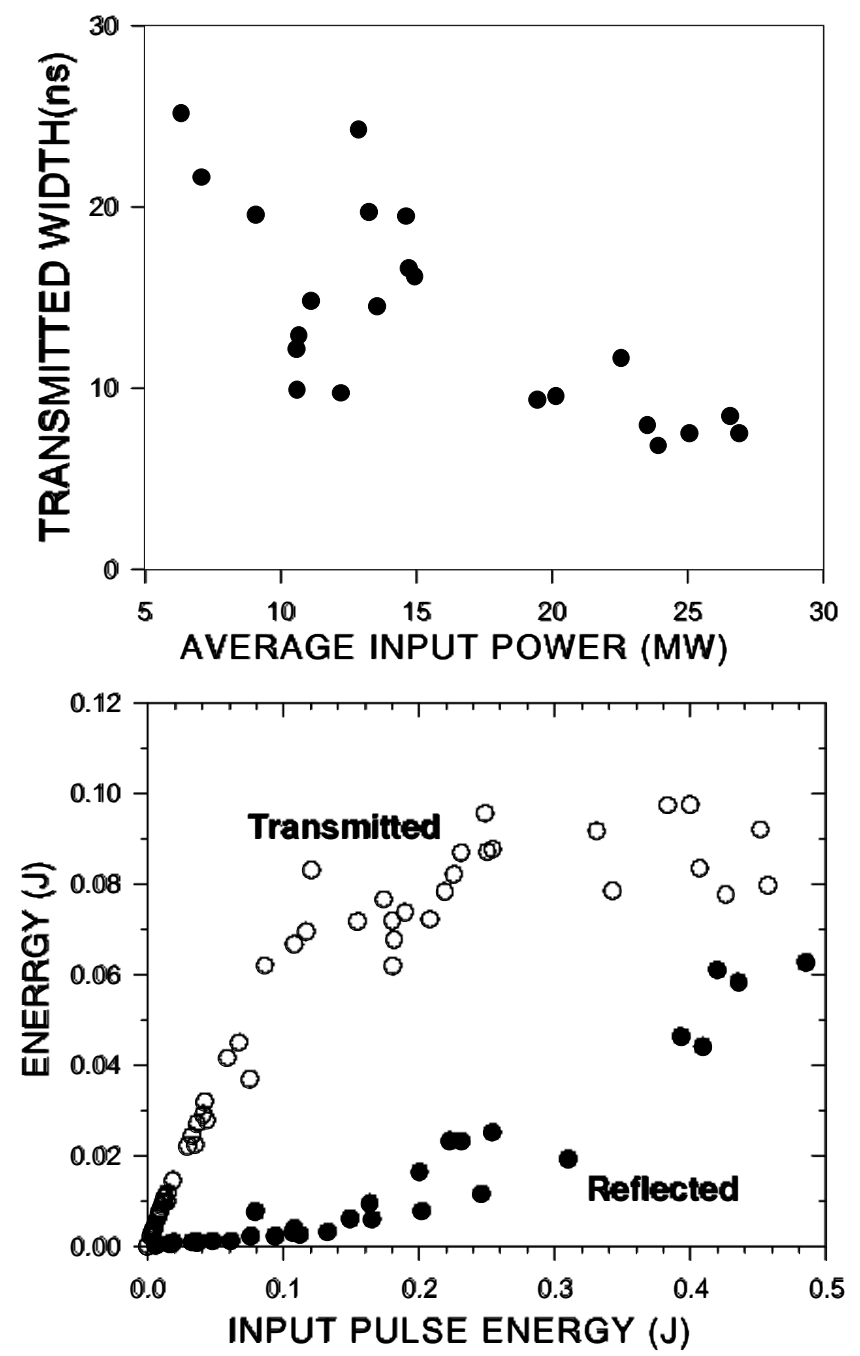

Figure 1: FWHM of the transmitted pulse vs. average input power, upper graph. Transmitted vs. input energy, lower.

\section{B. X-RAY and Visible Light Measurements}

Both light and X-ray induced photomultiplier signals were recorded for a range of r.f. input powers $(0.1$ MW to $30 \mathrm{MW}$ ) from the FEL. In Figure 2 we show plots of the maximum photomultiplier voltage from light and X-ray vs. average r.f. input power. Both light and X-ray generation starts around $1 \mathrm{MW}$ of input power and continues to rise exponentially with input power thereafter. To demonstrate this exponential growth we present the photomultiplier voltage plots on a logarithmic scale. The observed growth of both X-ray and visible light intensities begin around $1 \mathrm{MW}$ of input power which correlates well with the energy plots of Figure 1.
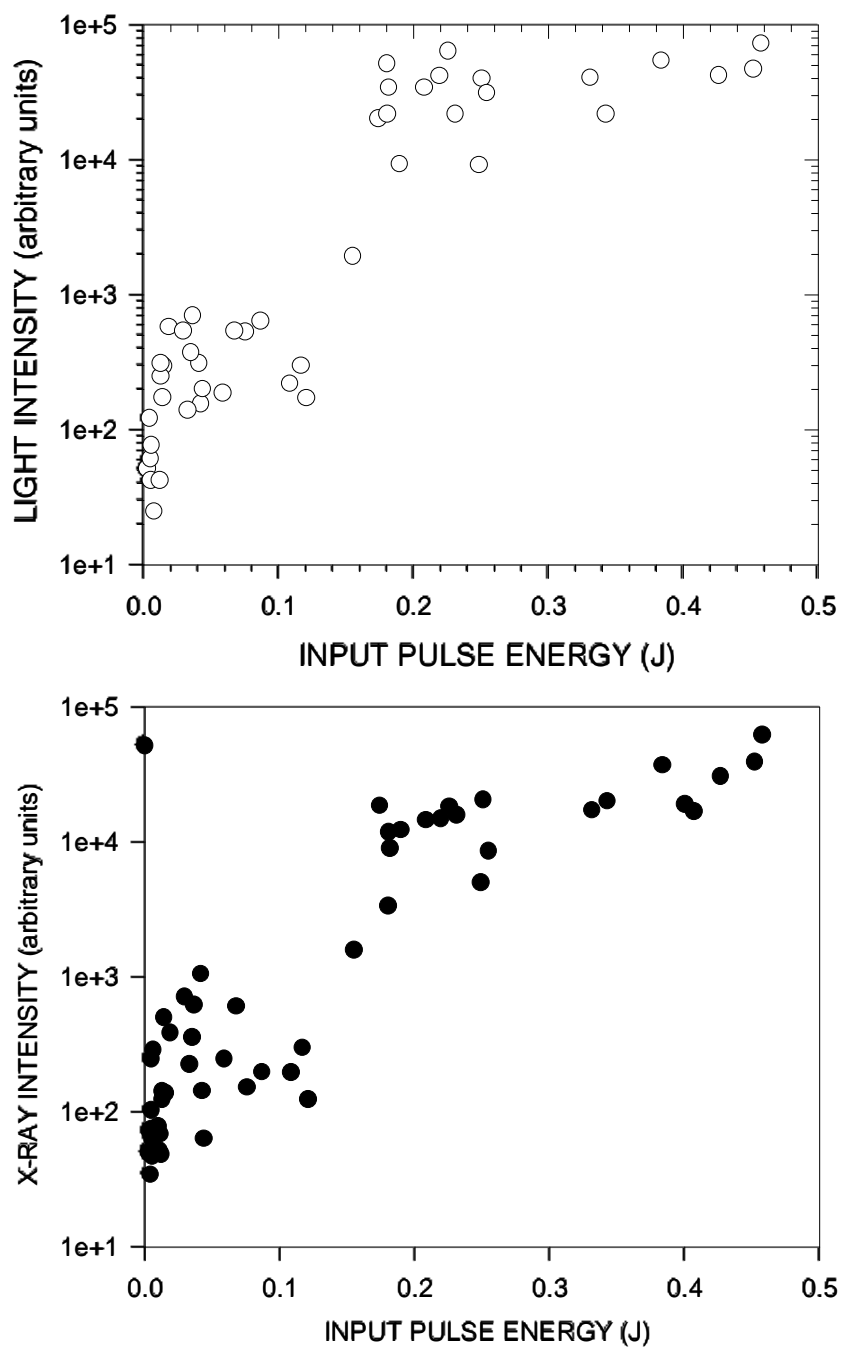

Figure 2: Light and X-ray induced photomultiplier signal vs. input energy, "log" scale.

\section{Operating at Different Frequency}

To show that there is an undesirable process in the input side coupler to the HGA we detune the FEL off the 0.5 $\mathrm{GHz}$ wide transmission band of the HGA to a new frequency $\mathrm{f}=33.682 \mathrm{GHz}$. The $33.682 \mathrm{GHz}$ radiation couples to an evanescent mode in the structure. The major portion of the pulse is reflected, with only about $10 \%$ of the incident energy is transmitted through the structure at this frequency. Thus the electric field gradients inside the 
structure itself are now much smaller than those in the input coupler. If the coupler causes the pulseshortening, the pulse shortening should be seen at the detuned frequency. Indeed, strong pulse-shortening was observed in reflected pulses. For high input energies $\sim 0.6 \mathrm{~J}$ the reflected energy constitutes only $20 \%$ of the input and the transmitted energy accounts for maybe another $2 \%$. A large fraction of the input energy is thus dissipated. The last two subsections have shown that there are strong indications that it is mainly in the input coupler that the pulse shortening takes place.

\section{Conditioning, Feasibility Test}

In this section we examine if the performance of the HGA improves with the number of shots, so that future conditioning could perhaps eliminate the pulse shortening. The estimated number of shots required for conditioning varies from a hundred thousand to several millions [6]. It did not seem possible to fully condition the HGA using the existing FEL (for it produces only one shot every two-three minutes and is manually operated). It is noted, however, that the rate of improvement was much greater during the first $5,000-10,000$ shots. This motivated the following test of the feasibility of conditioning in our HGA.

A power scan was made of the output and input pulses with the input power level ranging from 0.1 to 30 MW. An attempt to condition the structure was made with some 800 shots at power levels gradually increasing from 3$5 \mathrm{MW}$ to around $20 \mathrm{MW}$ were taken. The power scan was then repeated. Figure 3 shows plots of output vs. input energy before and after the conditioning.

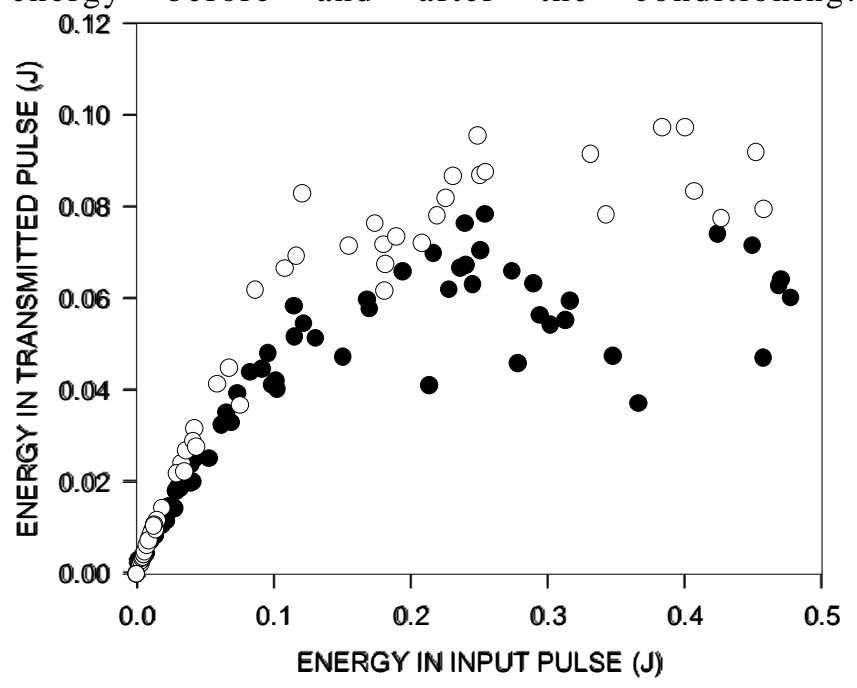

Figure 1: Transmitted vs. input energy. Black dots correspond to the data taken before 800 conditioning shots, hollow dots are after the attempt to condition..

The filled circles represent the data taken before conditioning, the hollow - after. One observes a definite improvement in transmission. There is evidence in favor of possibility of conditioning, provided a high enough power and repetition rate source at $33 \mathrm{GHz}$ is available.

\section{CONCLUSIONS}

The high power testing of a $33 \mathrm{GHz}$ HGA yielded the maximum accelerating gradient of $65 \mathrm{MeV} / \mathrm{m}$. A pulseshortening phenomenon was observed for power levels $>3$ MW. At the highest power level the output pulse length (about $5 \mathrm{~ns}$ ) was about one quarter of the input pulse length. Various tests suggest that these undesirable effects occur in the input coupler to the HGA. Light and X-ray production inside the HGA have been observed.

A study of feasibility of conditioning (limited by the low repetition rate of our FEL) suggest that conditioning is a likely candidate for improvement of the HGA power handling performance.

\section{REFERENCES}

[1] D.B. Hopkins, A.M. Sessler, and J.S. Wurtele. The Two-Beam Accelerator. Nucl. Inst. and Meth. in Phys. Res. 228, pp. 15-19, 1984.

[2] M.E. Conde and G. Bekefi. Experimental Study of a $33 \mathrm{GHz}$ FEL with a Reversed Axial Guide Magnetic Field. Phys. Rev. Lett., 67:3082, 1991.

[3] P. Volfbeyn, K. Ricci, B. Chen, G. Bekefi. Measurements of the Temporal and Spatial Phase Variations of a Pulsed Free Electron Laser Amplifier. IEEE Trans. on Plasma Science v. 22, no. 5, pp. 659-665, Oct 1994.

[4] Y. Baconnier et. al. The CERN Linear Collider Test Facility. Presented at the 1992 Linear Accelerator Conf. (Ottawa), CERN PS 92-49[LP].

[5] R. Bossart et. al. Performances Obtained with the CERN Linear Collider Test Facility. EPAC 94, Proc. v. 1, page $680,1994$.

[6] D.B. Hopkins and G.T. Conrad. Plans for Testing the 33.3 GHz High Gradient Accelerator. CP-49 Sept. 27, 1989. 